\title{
Bit Error Rate Performance of a MIMO-CDMA System Employing Parity-Bit-Selected Spreading in Frequency Nonselective Rayleigh Fading
}

\author{
Claude D'Amours ${ }^{1}$ and Adel Omar Dahmane ${ }^{2}$ \\ ${ }^{1}$ School of Electrical Engineering and Computer Science, University of Ottawa, Ottawa, Canada K1N 6N5 \\ ${ }^{2}$ Département de génie électrique et génie informatique, Université du Québec à Trois Rivières, Trois Rivières, Canada G9A 5H7
}

Correspondence should be addressed to Claude D’Amours, damours@site.uottawa.ca

Received 2 February 2011; Revised 15 May 2011; Accepted 27 June 2011

Academic Editor: Athanasios Panagopoulos

Copyright ( 2011 C. D’Amours and A. O. Dahmane. This is an open access article distributed under the Creative Commons Attribution License, which permits unrestricted use, distribution, and reproduction in any medium, provided the original work is properly cited.

\begin{abstract}
We analytically derive the upper bound for the bit error rate (BER) performance of a single user multiple input multiple output code division multiple access (MIMO-CDMA) system employing parity-bit-selected spreading in slowly varying, flat Rayleigh fading. The analysis is done for spatially uncorrelated links. The analysis presented demonstrates that parity-bit-selected spreading provides an asymptotic gain of $10 \log \left(N_{t}\right) \mathrm{dB}$ over conventional MIMO-CDMA when the receiver has perfect channel estimates. This analytical result concurs with previous works where the (BER) is determined by simulation methods and provides insight into why the different techniques provide improvement over conventional MIMO-CDMA systems.
\end{abstract}

\section{Introduction}

The object of much research in wireless communications is to enable users to transmit and receive at high and variable data rates to support the growing number of applications that involve such transfer of data [1]. Code Division Multiple Access (CDMA) systems employ spread spectrum (SS) technology and were developed for second and third generation $(2 \mathrm{G}, 3 \mathrm{G})$ wireless communications. For example, IS-95 and Wideband CDMA (WCDMA) systems are based on direct sequence SS techniques.

Multiple access interference (MAI) is present in CDMA systems due to the nonzero cross-correlation between the different users' spreading codes [2]. The MAI that each user's signal creates in all other users' signals results in increased bit error rate (BER). The overall system capacity is determined by the number of simultaneous transmitters that can be supported before the BER increases to an unacceptable level [3]. Much research presented in the literature has concentrated on making systems more power efficient as a means to increase the overall spectral efficiency of the CDMA system [4-6]. Other techniques, such as multiuser detection, have also been considered to increase the capacity of CDMA systems $[2,7,8]$.

Recent research has shown that combining DS-CDMA systems with Multiple Input Multiple Output (MIMO) techniques can achieve high gains in capacity, reliability and data transmission speed [9-14]. This is achieved by exploiting the spatial diversity made possible by multiple antennas at the transmitter and the receiver, allowing more degrees of freedom when the complex channel gains between different transmit and receive antenna pairs are sufficiently uncorrelated. MIMO-CDMA systems are also more robust to multiple access interference (MAI) than their single input single output (SISO) DS-CDMA counterparts. Currently, MIMO-CDMA is considered for many beyond 3G (B3G) applications [13, 14].

In [15], the concept of parity-bit-selected spreading for direct sequence spread spectrum (DS-SS) is introduced. In [16], the parity-bit-selected spreading technique is extended to code division multiple access (CDMA) systems using multiple input multiple output (MIMO) techniques. Also in [16], the parity-bit-selected spreading technique is modified to create the so-called permutation spreading technique. The 
permutation spreading technique combines spreading and space time coding to produce the effect of transmit diversity without retransmitting data from different antennas.

Compared to a conventional MIMO-CDMA system, which assigns a unique spreading waveform from a set of mutually orthogonal waveforms to each antenna, the MIMO-CDMA system employing either parity-bit-selected or permutation spreading provides significant power gains. This is demonstrated in [16] through the use of Monte Carlo simulations.

The BER performance of the aforementioned spreading techniques for MIMO-CDMA is also determined for systems encountering multiple access interference (MAI), spatial correlation, and/or channel estimation errors $[17,18]$. What is lacking in [16-18] is an analysis of the BER performance of MIMO-CDMA systems, employing these techniques. Such an analysis permits us to better understand why the new spreading techniques provide improvements over conventional MIMO-CDMA systems and this can, in turn, permit us to improve upon the new spreading techniques. In this paper, we provide, for the first time, an analytical expression for the upper bound of the BER for a MIMOCDMA system employing parity-bit-selected or permutation spreading.

\section{MIMO-CDMA System}

A MIMO-CDMA system has $N_{t}$ transmit and $N_{r}$ receive antennas. The serial data, whose bit rate is $R_{b}$, is converted into $N_{t}$ parallel data streams, each with bit rate $R_{b} / N_{t}$. The $i$ th data stream of user $m$ is spread by spreading waveform $w_{m i}(t)$, which is an antipodal signal with chip rate $R_{c}$ and is selected from a set of mutually orthogonal spreading waveforms $C_{m}=\left\{c_{m 1}(t), c_{m 2}(t), c_{m N}(t)\right\}$. In other words,

$$
\frac{1}{T} \int_{0}^{T} c_{m i}(t) c_{m j}(t) d t= \begin{cases}1, & i=j \\ 0, & i \neq j\end{cases}
$$

where $T=N_{t} / R_{b}$ is the signaling interval. In this paper, we consider the use of short spreading waveforms. A short spreading waveform is one whose duration is equal to one signaling interval. Thus on the interval $0 \leq t \leq T$, the $i$ th spreading waveform from the set $C_{m}$ can be described mathematically by

$$
c_{m i}(t)=\sum_{j=1}^{L} c_{m i}^{(j)} p\left(t-j T_{c}\right),
$$

where $c_{m i}^{(j)}= \pm 1$ is the $j$ th bipolar chip of the $m$ th user's $i$ th spreading waveform, $T_{c}=1 / R_{c}$ is the chip interval, $L$ is the number of chips in the spreading waveform and is given by $L=T / T_{c}=R_{c} N_{t} / R_{b}$, and $p(t)$ is the rectangular chip pulse shape given by

$$
p(t)= \begin{cases}1, & 0 \leq t \leq T_{c} \\ 0, & \text { otherwise }\end{cases}
$$

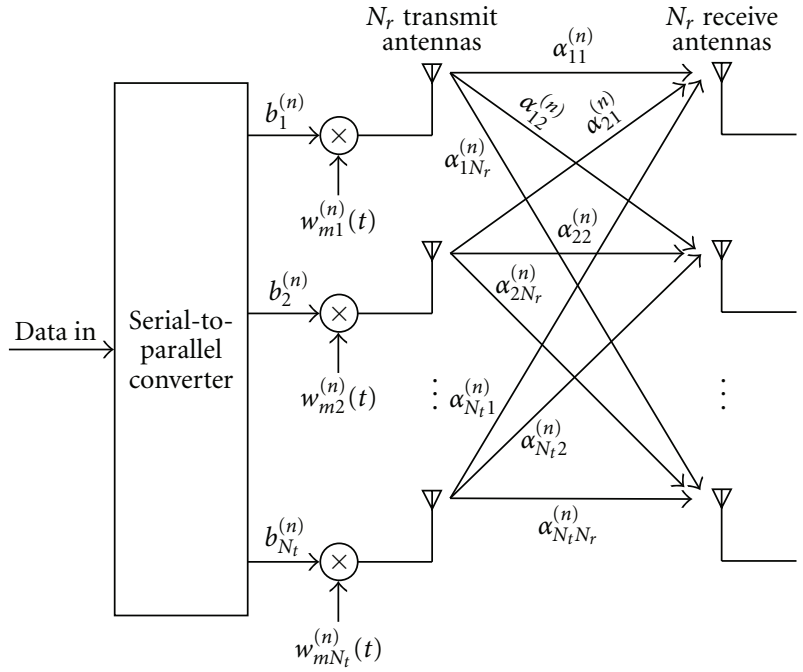

Figure 1: MIMO-CDMA transmitter and link gains.

Different users are assigned unique sets of spreading waveforms. Therefore, $C_{m} \cap C_{l}=\varnothing$ for $m \neq l$.

The transmitter, receive antennas and link gains are shown in Figure 1. On interval $(n-1) T \leq t \leq n T$ (signaling interval $n$ ), the data to be transmitted is $\mathbf{m}^{(n)}=$ $\left[m_{1}^{(n)}, m_{2}^{(n)}, \ldots, m_{N_{t}}^{(n)}\right]$, where $m_{k}^{(n)}$ is either 0 or 1 with equal probability and they are independent of one another. In this paper, we assume that binary phase shift keying (BPSK) modulation is used. Therefore, we define $\mathbf{b}^{(n)}=2 \mathbf{m}^{(n)}-$ $1=\left[b_{1}^{(n)}, b_{2}^{(n)}, \ldots, b_{N_{t}}^{(n)}\right]$, where $b_{k}^{(n)}$ for our baseband model is \pm 1 with equal probability and $E\left[b_{k}^{(n)} b_{g}^{(n)}\right]=0$, if $k \neq g$, where $E[\cdot]$ is the expectation operator. Each independent identically distributed (iid) bit to be transmitted on the $n$th signaling interval is assigned to a different transmit antenna, therefore, $b_{k}^{(n)}$ is transmitted on transmit antenna $k$. The $m$ th user's data bit, transmitted by transmit antenna $k$ on the $n$th signaling interval, is multiplied by spreading waveform $w_{m k}^{(n)}(t)$. The complex channel gain between transmit antenna $k$ and receive antenna $j$ on the $n$th signaling interval is $\alpha_{i j}^{(n)}$.

The receiver for MIMO-CDMA is shown in Figure 2. Here, the signal received by each receive antenna is correlated with each spreading waveform, and the contributions from the different antennas are combined according to the spreading technique used. The $k$ th matched filter output on receive antenna $j$ and signaling interval $n$ is $u_{j k}^{(n)}$. The expressions for these decision variables and how they are combined depend on the spreading method used by the transmitter.

\section{Parity-Bit-Selected Spreading}

Parity-bit-selected spreading for spread spectrum systems is introduced in [1]. The technique therein is modified slightly for MIMO-CDMA. the vector $\mathbf{m}^{(n)}$ is input to a parity-bit generator. The parity-bit generator produces a vector $\mathbf{p}^{(n)}=$ $\left[p_{1}^{(n)}, p_{2}^{(n)}, \ldots, p_{N}^{(n)}\right]$, where $N$ is the number of spreading codes used and is given by $N=2^{\text {length(p) }}$. There are as many parity 


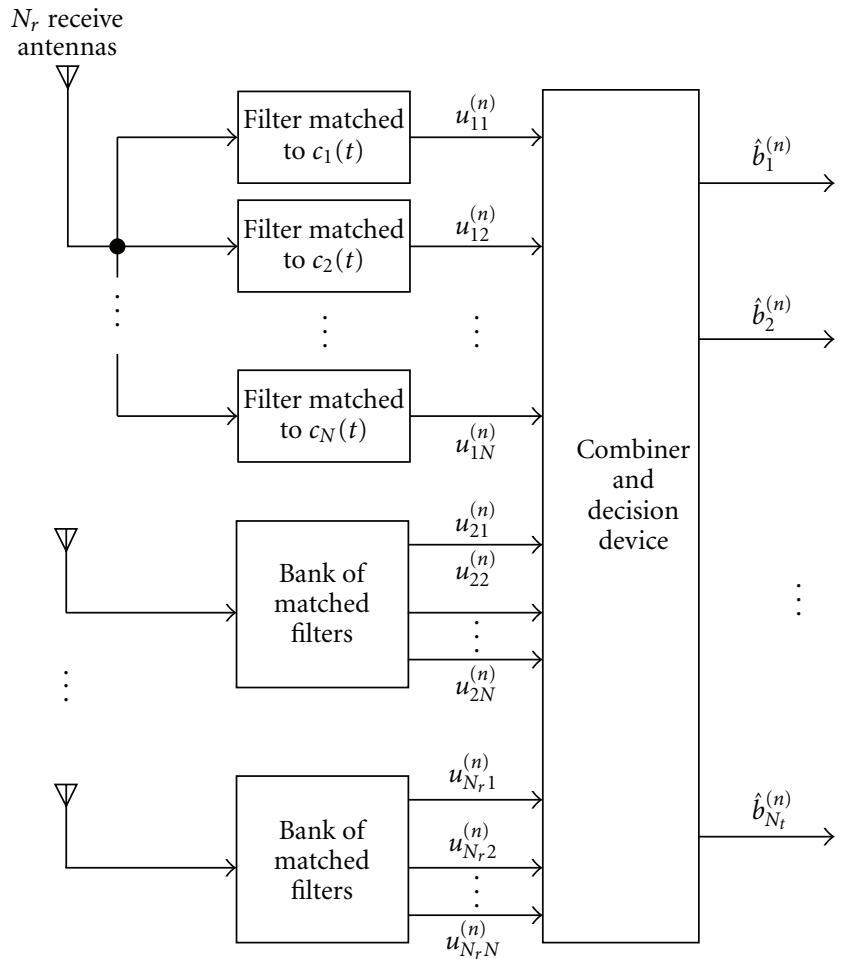

Figure 2: MIMO-CDMA receiver.

bit patterns as there are spreading waveforms in each user's set. Each spreading waveform is assigned to one of the parity bit vectors. Therefore, if $\mathbf{p}^{(n)}=\mathbf{p}_{x}$, then

$$
w_{m i}^{(n)}(t)=c_{m x}(t-n T) \text { for } i=1,2, \ldots, N_{t} .
$$

For the spreading strategy described by (4), the decision variables of Figure 2 are given by

$$
u_{j k}^{(n)}= \begin{cases}\sum_{i=1}^{N_{t}}\left(\sqrt{E_{b}} b_{i}^{(n)} \alpha_{i j}^{(n)}\right)+n_{j k}^{(n)}, & k=x, \\ n_{j k}^{(n)}, & k \neq x,\end{cases}
$$

where $E_{b}$ is the received energy per bit.

$$
\text { Let } \mathbf{u}^{(n)}=\left[u_{11}^{(n)}, u_{12}^{(n)}, \ldots, u_{1 N}^{(n)}, u_{21}^{(n)}, \ldots, u_{2 N}^{(n)}, \ldots, u_{N_{r} N}^{(n)}\right]
$$
which is a $1 \times N_{r} N$ vector. The $x$ th channel gain matrix, $\mathbf{H}_{x}^{(n)}$ is an $N_{t} \times N_{r} N$ matrix, is defined as

$$
\begin{gathered}
\mathbf{H}_{x}^{(n)}=\left[\mathbf{0}_{N_{t},(x-1)} \mathbf{h}_{1}^{(n)} \mathbf{0}_{N_{t},(N-1)} \mathbf{h}_{2}^{(n)} \mathbf{0}_{N_{t},(N-1)},\right. \\
\left.\mathbf{h}_{3}^{(n)} \cdots \mathbf{h}_{N_{r}}^{(n)} \mathbf{0}_{N_{t},(N-x)}\right]
\end{gathered}
$$

where $\mathbf{0}_{a, b}$ is an $a \times b$ all zero matrix and $\mathbf{h}_{i}^{(n)}=$ $\left[\alpha_{1 i}^{(n)}, \alpha_{2 i}^{(n)}, \ldots, \alpha_{N_{t} i}^{(n)}\right]^{T}$. The channel matrix used on signaling interval $n$ depends on the spreading waveform used during that interval. For example, if the transmitter employs $c_{m 1}(t-$ $n T)$, then $\mathbf{H}_{1}^{(n)}$ is the appropriate channel gain matrix to use.

We can now express $\mathbf{u}^{(n)}$ as

$$
\mathbf{u}^{(n)}=\sqrt{E_{b}} \mathbf{b}^{(n)} \mathbf{H}_{b}^{(n)}+\mathbf{n}^{(n)},
$$

TABLE 1: The allocation of spreading waveforms in parity bit selected spreading technique for $N_{t}=4$.

\begin{tabular}{lcc}
\hline $\begin{array}{l}\text { Messages } \\
\mathbf{m}^{(n)}\end{array}$ & $\begin{array}{c}\text { Spreading waveform } \\
\text { used }\end{array}$ & $\begin{array}{c}\text { Channel gain matrix } \\
\text { used in }(7) \text { and }(8)\end{array}$ \\
\hline 0000 & $c_{m 1}(t-n T)$ & $\mathbf{H}_{1}^{(n)}$ \\
1111 & $c_{m 2}(t-n T)$ & $\mathbf{H}_{2}^{(n)}$ \\
\hline 0001 & $c_{m 3}(t-n T)$ & $\mathbf{H}_{3}^{(n)}$ \\
\hline 1110 & $c_{m 4}(t-n T)$ & $\mathbf{H}_{4}^{(n)}$ \\
\hline 0010 & $c_{m 5}(t-n T)$ & $\mathbf{H}_{5}^{(n)}$ \\
\hline 1101 & $c_{m 6}(t-n T)$ & $\mathbf{H}_{6}^{(n)}$ \\
\hline 0011 & $c_{m 7}(t-n T)$ & $\mathbf{H}_{7}^{(n)}$ \\
\hline 1100 & $c_{m 8}(t-n T)$ & $\mathbf{H}_{8}^{(n)}$ \\
\hline 0101 & &
\end{tabular}

where $\mathbf{H}_{b}^{(n)}$ is the channel matrix associated with data vector $\mathbf{b}^{(n)}$ and $\mathbf{n}^{(n)}=\left[n_{11}^{(n)}, n_{12}^{(n)}, \ldots, n_{21}^{(n)}, \ldots, n_{N_{r} N}^{(n)}\right]$ is a $1 \times N_{r} N$ noise vector. The elements of $\mathbf{n}^{(n)}$ are uncorrelated zero mean complex Gaussian random variables with variance $\sigma_{n}^{2}$.

In [15], a suboptimum detection scheme is presented in which the detector determines which spreading code has been used and then detects the data only by observing the outputs matched to that code and comparing them to the possible messages associated with that code. In this paper, maximum likelihood (ML) detection is considered. The receiver does not try to determine which code has been used. It compares $\mathbf{u}^{(n)}$ to all possible receive vectors $\sqrt{E_{b}} \mathbf{b}_{i} \mathbf{H}_{b_{i}}^{(n)}$, where $\mathbf{H}_{b_{i}}$ is the channel matrix associated with transmitted message $\mathbf{b}_{i}$. The receiver selects $\widehat{\mathbf{b}}^{(n)}$ as the message vector that minimizes the square of the Euclidean distance as shown in:

$$
\widehat{\mathbf{b}}^{(n)}=\min _{\mathbf{b}_{i} \in B}\left\|u^{(n)}-\sqrt{E_{b}} \mathbf{b}_{i} \mathbf{H}_{b_{i}}^{(n)}\right\|^{2}
$$

where $B$ is the set of all possible transmitted data vectors.

We consider the four transmit antenna case of [2]. Table 1 shows the allocation of spreading waveforms to message vectors as well as the corresponding channel gain matrix that is used in (7) and (8).

From Table 1, we see that the two message vectors m $=0000$ and $\mathbf{m}=1111$ (which correspond to $\mathbf{b}=-1-1$ $-1-1$ and $\mathbf{b}=1111$ ) form a subset of all possible binary message vectors of length 4 . This subset is spread using spreading waveform $c_{m 1}(t-n T)$. The messages associated with other spreading waveforms are simply cosets of the subset $\{0000,1111\}$. Therefore, we can state that each coset is assigned a unique spreading waveform.

As previously mentioned, ML detection is used in this paper. Therefore, the squared Euclidean distance between 
$\mathbf{u}^{(n)}$ and each of the vectors in Table 1 is computed, and the message vector corresponding to the smallest squared distance is selected as the most likely transmitted message. In terms of BER performance, this is equivalent to correlating $\mathbf{u}^{(n)}$ to each of the vectors in Table 1 and selecting the one with the highest correlation value. The correlation technique is in fact the least computationally complex method as many terms in the vectors in Table 1 are 0 . However, if additional coding is used, the Euclidean distances are useful for calculating log likelihood ratios for the decoder.

\section{BER Performance of MIMO-CDMA Using Parity-Bit-Selected Spreading}

In CDMA systems, bit error rate (BER) performance is a very important parameter. Not only does BER determine the quality of transmission, but also does it determine the amount of data that can be transmitted per unit of bandwidth. As every user contributes to the interference levels at the receiver, the BER of each user increases as more users access the channel. Thus, the maximum number of users is determined by the amount of interference that can be tolerated.

We will determine the BER performance of MIMOCDMA employing parity-bit-selected spreading analytically using the following assumptions.

(1) The MIMO-CDMA system under consideration uses four transmit antennas and eight spreading waveforms as in [2] and as detailed in Table 1.

(2) The fading process is assumed to be frequency nonselective. In other words, the multipath spread is zero and there is no channel induced intersymbol interference (ISI).

(3) The channel gains are independent slowly varying circularly-symmetric complex Gaussian random variables with zero mean and unit variance.

(4) The channel gains are known to the receiver, thus coherent detection is performed.

When determining the BER of communication systems operating in Rayleigh fading channel with diversity order $L$, typically we are required to integrate a Q-function multiplied by the probability density function (pdf) of the energy per bit to noise spectral density ratio $\left(E_{b} / N_{o}\right)$. The pdf of $E_{b} / N_{o}$ is a chi-square distribution with $2 L$ degrees of freedom. The result of this integral takes to form of $p(L, x)[19]$ which is shown in:

$$
p(L, x)=\left(\frac{1-\mu}{2}\right) \sum_{k=0}^{L L-1}\left(\begin{array}{c}
L-1+k \\
k
\end{array}\right)\left(\frac{1+\mu}{2}\right)^{k},
$$

where $\mu=\sqrt{x /(x+1)}$.

4.1. Single Receive Antenna, $N_{r}=1$. We start by assuming that there is only one receive antenna. Thus, the received vector is

$$
\mathbf{u}^{(n)}=\left[u_{11}^{(n)}, u_{12}^{(n)}, \ldots, u_{1 N}^{(n)}\right]
$$

TABLE 2: The received vectors for the parity-bit-selected spreading technique for $N_{t}=4$ and $N_{r}=1$.

\begin{tabular}{lc}
\hline $\begin{array}{c}\text { Messages } \\
\mathbf{m}^{(n)}\end{array}$ & $\begin{array}{c}\text { Received vector } \\
\mathbf{u}^{(n)}\end{array}$ \\
\hline $0: 0000$ & {$\left[-\sqrt{E_{b}}\left(\alpha_{11}^{(n)}+\alpha_{21}^{(n)}+\alpha_{31}^{(n)}+\alpha_{41}^{(n)}\right), 0,0,0,0,0,0,0\right]$} \\
$15: 1111$ & {$\left[\sqrt{E_{b}}\left(\alpha_{11}^{(n)}+\alpha_{21}^{(n)}+\alpha_{31}^{(n)}+\alpha_{41}^{(n)}\right), 0,0,0,0,0,0,0\right]$} \\
$1: 0001$ & {$\left[0,-\sqrt{E_{b}}\left(\alpha_{11}^{(n)}+\alpha_{21}^{(n)}+\alpha_{31}^{(n)}-\alpha_{41}^{(n)}\right), 0,0,0,0,0,0\right]$} \\
$14: 1110$ & {$\left[0, \sqrt{E_{b}}\left(\alpha_{11}^{(n)}+\alpha_{21}^{(n)}+\alpha_{31}^{(n)}-\alpha_{41}^{(n)}\right), 0,0,0,0,0,0\right]$} \\
$2: 0010$ & {$\left[0,0,-\sqrt{E_{b}}\left(\alpha_{11}^{(n)}+\alpha_{21}^{(n)}-\alpha_{31}^{(n)}+\alpha_{41}^{(n)}\right), 0,0,0,0,0\right]$} \\
$13: 1101$ & {$\left[0,0, \sqrt{E_{b}}\left(\alpha_{11}^{(n)}+\alpha_{21}^{(n)}-\alpha_{31}^{(n)}+\alpha_{41}^{(n)}\right), 0,0,0,0,0\right]$} \\
$3: 0011$ & {$\left[0,0,0,-\sqrt{E_{b}}\left(\alpha_{11}^{(n)}+\alpha_{21}^{(n)}-\alpha_{31}^{(n)}-\alpha_{41}^{(n)}\right), 0,0,0,0\right]$} \\
$12: 1100$ & {$\left[0,0,0, \sqrt{E_{b}}\left(\alpha_{11}^{(n)}+\alpha_{21}^{(n)}-\alpha_{31}^{(n)}-\alpha_{41}^{(n)}\right), 0,0,0,0\right]$} \\
$4: 0100$ & {$\left[0,0,0,0,-\sqrt{E_{b}}\left(\alpha_{11}^{(n)}-\alpha_{21}^{(n)}+\alpha_{31}^{(n)}+\alpha_{41}^{(n)}\right), 0,0,0\right]$} \\
$11: 1011$ & {$\left[0,0,0,0, \sqrt{E_{b}}\left(\alpha_{11}^{(n)}-\alpha_{21}^{(n)}+\alpha_{31}^{(n)}+\alpha_{41}^{(n)}\right), 0,0,0\right]$} \\
$5: 0101$ & {$\left[0,0,0,0,0,-\sqrt{E_{b}}\left(\alpha_{11}^{(n)}-\alpha_{21}^{(n)}+\alpha_{31}^{(n)}-\alpha_{41}^{(n)}\right), 0,0\right]$} \\
$10: 1010$ & {$\left[0,0,0,0,0, \sqrt{E_{b}}\left(\alpha_{11}^{(n)}-\alpha_{21}^{(n)}+\alpha_{31}^{(n)}-\alpha_{41}^{(n)}\right), 0,0\right]$} \\
$6: 0110$ & {$\left[0,0,0,0,0,0,-\sqrt{E_{b}}\left(\alpha_{11}^{(n)}-\alpha_{21}^{(n)}-\alpha_{31}^{(n)}+\alpha_{41}^{(n)}\right), 0\right]$} \\
$9: 1001$ & {$\left[0,0,0,0,0,0, \sqrt{E_{b}}\left(\alpha_{11}^{(n)}-\alpha_{21}^{(n)}-\alpha_{31}^{(n)}+\alpha_{41}^{(n)}\right), 0\right]$} \\
$7: 0111$ & {$\left[0,0,0,0,0,0,0,-\sqrt{E_{b}}\left(\alpha_{11}^{(n)}-\alpha_{21}^{(n)}-\alpha_{31}^{(n)}-\alpha_{41}^{(n)}\right)\right]$} \\
$8: 1000$ & {$\left[0,0,0,0,0,0,0, \sqrt{E_{b}}\left(\alpha_{11}^{(n)}-\alpha_{21}^{(n)}-\alpha_{31}^{(n)}-\alpha_{41}^{(n)}\right)\right]$} \\
\hline
\end{tabular}

In the absence of noise, the set of all received vectors form an 8 dimensional constellation. The points of the constellation are given in Table 2 . The variable $E_{b}$ is the average energy per bit.

For ML detection, the probability of symbol error, as well as the BER, can be determined by the Euclidean distance between the constellation points. Let us assume that the transmitted message is message $0(0000)$. The probability that message 15 (1111) is detected, given that message 0 is transmitted, is a function of the distance between their constellation points, $d_{15,0}$. This distance is given by

$$
\begin{aligned}
d_{15,0}^{2} & =\left\|\mathbf{u}_{1111}-\mathbf{u}_{0000}\right\|^{2} \\
& =4 E_{b}\left|\alpha_{11}^{(n)}+\alpha_{21}^{(n)}+\alpha_{31}^{(n)}+\alpha_{41}^{(n)}\right|^{2} .
\end{aligned}
$$

We let $X=\alpha_{11}^{(n)}+\alpha_{21}^{(n)}+\alpha_{31}^{(n)}+\alpha_{41}^{(n)}$ and $Z=|X|^{2}$. Because of assumption (3) above, $X$ is a zero mean complex Gaussian random variable with variance 4 . Thus, $Z$ has a chi-square probability density function (pdf) with 2 degrees of freedom [19]. It has a mean of 4. Thus, the pdf of $Z$ is given by

$$
f_{Z}(z)=\frac{1}{4} e^{-z / 4}, \quad 0<z<\infty .
$$

The probability of incorrectly detecting message 15 when message 0 is transmitted is

$$
P(15 \mid 0)=\frac{1}{2} \operatorname{erfc}\left(\sqrt{\frac{d_{15,0}^{2}}{4 N_{o}}}\right)=\frac{1}{2} \operatorname{erfc}\left(\sqrt{\frac{E_{b} Z}{N_{o}}}\right),
$$

where $N_{o}$ is the single-sided noise spectral density. We see that $P(15 \mid 0)$ is a function of the random variable $Z$. Thus, $E[P(15 \mid 0)]=p_{15 \mid 0}$ is found by multiplying $P(15 \mid 0)$ by 
the pdf of $Z$ and integrating from 0 to $\infty$. This is shown in [19] to be

$$
\begin{aligned}
p_{15 \mid 0} & =\int_{0}^{\infty} \frac{1}{8} \operatorname{erfc}\left(\sqrt{\frac{E_{b} Z}{N_{o}}}\right) e^{-z / 4} d z \\
& =\frac{1}{2}\left[1-\sqrt{\frac{4 \gamma_{b}}{1+4 \gamma_{b}}}\right]=p\left(1,4 \gamma_{b}\right),
\end{aligned}
$$

where $\gamma_{b}=E_{b} / N_{o}$ is the average energy per bit to noise spectral density ratio.

Next we consider the probability that message $1(0001)$ is detected when message 0 is transmitted. The distance $d_{1,0}$ is given by

$$
\begin{aligned}
d_{1,0}^{2}= & \left\|\mathbf{u}_{0001}-\mathbf{u}_{0000}\right\|^{2} \\
= & E_{b}\left|\alpha_{11}^{(n)}+\alpha_{21}^{(n)}+\alpha_{31}^{(n)}+\alpha_{41}^{(n)}\right|^{2} \\
& +E_{b}\left|\alpha_{11}^{(n)}+\alpha_{21}^{(n)}+\alpha_{31}^{(n)}-\alpha_{41}^{(n)}\right|^{2} .
\end{aligned}
$$

It can be shown that $|A+B|^{2}+|A-B|^{2}=2|A|^{2}+2|B|^{2}$, thus $d_{1,0}^{2}$ becomes

$$
d_{1,0}^{2}=2 E_{b}\left(\left|\alpha_{11}^{(n)}+\alpha_{21}^{(n)}+\alpha_{31}^{(n)}\right|^{2}+\left|\alpha_{41}^{(n)}\right|^{2}\right)
$$

Let $Y_{A}=\alpha_{11}^{(n)}+\alpha_{21}^{(n)}+\alpha_{31}^{(n)}$ and $Y_{B}=\alpha_{41}^{(n)}$. Also, let $W_{A}=$ $\left|Y_{A}\right|^{2}$ and $W_{B}=\left|Y_{B}\right|^{2}$. Finally, let $W=W_{A}+W_{B}$. Although $W$ has the same mean as $Z$, it does not have the same higher order statistics, nor does it have the same pdf. The pdfs of $W_{A}$ and $W_{B}$ are

$$
\begin{gathered}
f_{W_{A}}(w)=\frac{1}{3} e^{-w / 3}, \quad 0<w<\infty, \\
f_{W_{B}}(w)=e^{-w}, \quad 0<w<\infty .
\end{gathered}
$$

Since the channel gains are i.i.d., $W_{A}$ and $W_{B}$ are independent. Thus the pdf of $W=W_{A}+W_{B}$ is given by

$$
\begin{aligned}
f_{W}(w) & =f_{W_{A}}(w) * f_{W_{B}}(w) \\
& =\frac{1}{2} e^{-w / 3}-\frac{1}{2} e^{-w}, \quad 0<w<\infty,
\end{aligned}
$$

where $*$ denotes convolution. It can be seen that $f_{W}(w)$ is a weighted sum of chi-square distributions with two degrees of freedom. Therefore, the expected probability of detecting message 1 when message 0 is transmitted is [19]

$$
\begin{aligned}
p_{1 \mid 0} & =\int_{0}^{\infty} \frac{1}{2} \operatorname{erfc}\left(\sqrt{\frac{E_{b} W}{2 N_{o}}}\right) f_{W}(w) d w \\
& =\frac{3}{4}\left[1-\sqrt{\frac{3 \gamma_{b}}{2+3 \gamma_{b}}}\right]-\frac{1}{4}\left[1-\sqrt{\frac{\gamma_{b}}{2+\gamma_{b}}}\right] \\
& =\frac{3}{2} p\left(1, \frac{3}{2} \gamma_{b}\right)-\frac{1}{2} p\left(1, \frac{1}{2} \gamma_{b}\right) .
\end{aligned}
$$

We can show that distances between messages 2, 4, 7, 8, 11,13 , and 14 and message 0 all have the same statistics as the distance between message 1 and message 0 . Therefore $p_{1 \mid 0}=p_{2 \mid 0}=p_{4 \mid 0}=p_{7 \mid 0}=p_{8 \mid 0}=p_{1|| 0}=p_{13 \mid 0}=p_{14 \mid 0}$. by

The distance between message 3 and message 0 is given

$$
\begin{aligned}
d_{3,0}^{2}= & \left\|\mathbf{u}_{0011}-\mathbf{u}_{0000}\right\|^{2} \\
= & E_{b}\left|\alpha_{11}^{(n)}+\alpha_{21}^{(n)}+\alpha_{31}^{(n)}+\alpha_{41}^{(n)}\right|^{2} \\
& +E_{b}\left|\alpha_{11}^{(n)}+\alpha_{21}^{(n)}-\alpha_{31}^{(n)}-\alpha_{41}^{(n)}\right|^{2} \\
= & 2 E_{b}\left(\left|\alpha_{11}^{(n)}+\alpha_{21}^{(n)}\right|^{2}+\left|\alpha_{31}^{(n)}+\alpha_{41}^{(n)}\right|^{2}\right) .
\end{aligned}
$$

Here, we let $V=V_{A}+V_{B}$, where $V_{A}=\left|\alpha_{11}^{(n)}+\alpha_{21}^{(n)}\right|^{2}$ and $V_{B}=\left|\alpha_{31}^{(n)}+\alpha_{41}^{(n)}\right|^{2}$ are independent chi-square distributed random variables. The pdfs of $V_{A}$ and $V_{B}$ are

$$
f_{V_{A}}(v)=f_{V_{B}}(v)=\frac{1}{2} e^{-v / 2}, \quad 0<v<\infty .
$$

The pdf of $V$ is $f_{V}(v)=f_{V_{A}}(v) * f_{V_{B}}(v)$, which is

$$
f_{V}(v)=\frac{1}{4} v e^{-v / 2}, \quad 0<v<\infty,
$$

which is a chi-square distribution with 4 degrees of freedom. The expected probability of detecting message 3 when message 0 is transmitted is [19]

$$
p_{3 \mid 0}=\int_{0}^{\infty} \frac{1}{2} \operatorname{erfc}\left(\sqrt{\frac{\gamma_{b} V}{2}}\right) f_{V}(v) d v=p\left(2, \gamma_{b}\right)
$$

It can be shown that the distance between messages 5 , $6,9,10$, and 12 and message 0 have the same statistics as the distance between message 3 and message 0 . Thus, $p_{3 \mid 0}=$ $p_{5 \mid 0}=p_{6 \mid 0}=p_{9 \mid 0}=p_{10 \mid 0}=p_{12 \mid 0}$.

Using a union bound, we can estimate the probability of detection error when message 0 is transmitted as

$$
\begin{aligned}
P(E \mid 0)< & \sum_{i=1}^{15} p_{i \mid 0} \\
< & p\left(1,4 \gamma_{b}\right)+8 p\left(1, \frac{3}{2} \gamma_{b}\right) \\
& -4 p\left(1, \frac{1}{2} \gamma_{b}\right)+6 p\left(2, \gamma_{b}\right) .
\end{aligned}
$$

We can show that the probability of detection error is independent of which message was transmitted; therefore, $P(E \mid 0)=P(E)$. Similarly, the probability of bit error is independent of the transmitted message; therefore, we can use the conditional expressions that we derived previously to determine an upper bound. It is given by

$$
P_{b}<p\left(1,4 \gamma_{b}\right)+4 p\left(1, \frac{3}{2} \gamma_{b}\right)-2 p\left(1, \frac{1}{2} \gamma_{b}\right)+3 p\left(2, \gamma_{b}\right) .
$$


4.2. Multiple Receive Antennas, $N_{r}>1$. In the single user case, the use of multiple receive antennas provides BER improvement through receive diversity. The received vector is a $1 \times 8 N_{r}$ vector. As an example, $\mathbf{u}_{0000}$ is

$$
\mathbf{u}_{0000}=\left[\mathbf{u}_{0000_{1}}\left|\mathbf{u}_{0000_{2}}\right| \cdots \mid \mathbf{u}_{0000_{N_{r}}}\right],
$$

where $\mathbf{u}_{0000_{i}}=\left[-\sqrt{E_{b} / N_{r}}\left(\alpha_{1 i}^{(n)}+\alpha_{2 i}^{(n)}+\alpha_{3 i}^{(n)}+\alpha_{4 i}^{(n)}\right), 0, \ldots, 0\right]$.

The probability that message 15 is detected when message 0 is sent is given by

$$
p_{15 \mid 0}=\int_{0}^{\infty} \frac{1}{2} \operatorname{erfc}\left(\sqrt{\frac{\gamma_{b} Z^{\left(N_{r}\right)}}{N_{r}}}\right) f_{Z^{\left(N_{r}\right)}}(z) d z,
$$

where $Z^{\left(N_{r}\right)}=Z_{1}+Z_{2}+\cdots+Z_{N_{r}}$. Each of the $Z_{i}$ 's has a pdf that is given by (12). Assuming no spatial correlation, the $Z_{i}$ s are mutually independent; therefore $f_{Z^{\left(N_{r}\right)}}(z)$ is the convolution of (12) with itself $N_{r}-1$ times. Thus, as shown in [19], $f_{Z}^{\left(N_{r}\right)}(z)$ is

$$
f_{Z^{\left(N_{r}\right)}}(z)=\frac{1}{\left(N_{r}-1\right) ! 4^{N_{r}}} z^{N_{r}-1} e^{-z / 4}, \quad 0<z<\infty,
$$

which is a chi-square distribution with $2 N_{r}$ degrees of freedom. Therefore, from [19], we can show that $p_{15 \mid 0}$ is

$$
p_{15 \mid 0}=p\left(N_{r}, \frac{4 \gamma_{b}}{N_{r}}\right) .
$$

The probability of detecting message 3 when message 0 is transmitted is given by

$$
p_{3 \mid 0}=\int_{0}^{\infty} \frac{1}{2} \operatorname{erfc}\left(\sqrt{\frac{\gamma_{b} V^{\left(N_{r}\right)}}{N_{r}}}\right) f_{V^{\left(N_{r}\right)}}(v) d v,
$$

where $V^{\left(N_{r}\right)}=V_{1}+V_{2}+\cdots+V_{N_{r}}$, and each $V_{i}$ has distribution $f_{V_{i}}(v)$ that is given by $(22)$. Therefore, $f_{V^{\left(N_{r}\right)}}(v)$ is the convolution of (22) with itself $N_{r}-1$ times. We can show that $f_{V^{\left(N_{r}\right)}}(v)$ is given by

$$
f_{V^{\left(N_{r}\right)}}(z)=\frac{1}{\left(2 N_{r}-1\right) ! 2^{2 N_{r}}} v^{2 N_{r}-1} e^{-v / 2}, \quad 0<v<\infty,
$$

which is a chi-square distribution with $4 N_{r}$ degrees of freedom. Therefore, $p_{3 \mid 0}$ is

$$
p_{3 \mid 0}=p\left(2 N_{r}, \frac{\gamma_{b}}{N_{r}}\right)
$$

Lastly, the probability $p(1 \mid 0)$ is given by

$$
p_{1 \mid 0}=\int_{0}^{\infty} \frac{1}{2} \operatorname{erfc}\left(\frac{\gamma_{b} W^{\left(N_{r}\right)}}{2 N_{r}}\right) f_{W^{\left(N_{r}\right)}}(w) d w,
$$

where $W^{\left(N_{r}\right)}=W_{1}+W_{2}+\cdots+W_{N_{r}}$. Each $W_{i}$ has distribution $f_{W_{i}}(w)$ given by (18). To find the pdf of $W^{\left(N_{r}\right)}$ we must convolve (18) with itself $N_{r}-1$ times. Therefore:

$$
\begin{aligned}
f_{W^{\left(N_{r}\right)}}(w)= & \frac{1}{4} w e^{-w / 3}+\frac{1}{4} w e^{-w} \\
& -\frac{3}{4} e^{-w / 3}+\frac{3}{4} e^{-w}, \quad 0<w<\infty
\end{aligned}
$$

for $N_{r}=2$. For $N_{r}=3$ it is given by:

$$
\begin{aligned}
f_{W^{\left(N_{r}\right)}}(w)= & \left(e^{-\mathrm{w} / 3}-e^{-w}\right)\left(\frac{1}{16} w^{2}+\frac{27}{16}\right) \\
& -\frac{9}{16} w\left(e^{-\mathrm{w} / 3}+e^{-w}\right), \quad 0<w<\infty .
\end{aligned}
$$

For $N_{r}=4$ the pdf is given by:

$$
\begin{aligned}
f_{W^{\left(N_{r}\right)}}(w)= & \left(e^{-w / 3}+e^{-w}\right)\left(\frac{1}{96} w^{3}+\frac{45}{32} w\right)-\left(e^{-w / 3}-e^{-w}\right) \\
& \times\left(\frac{3}{16} w^{2}+\frac{135}{32}\right), \quad 0<w<\infty .
\end{aligned}
$$

Using these pdfs and the results in [19], we can find $p_{1 \mid 0}$. For $N_{r}=2, p_{1 \mid 0}$ is given by:

$$
\begin{aligned}
p_{1 \mid 0}= & \frac{9}{4} p\left(2, \frac{3 \gamma_{b}}{4}\right)+\frac{1}{4} p\left(2, \frac{\gamma_{b}}{4}\right)-\frac{9}{4} p\left(1, \frac{3 \gamma_{b}}{4}\right) \\
& +\frac{3}{4} p\left(1, \frac{\gamma_{b}}{4}\right) .
\end{aligned}
$$

For $N_{r}=3, p_{1 \mid 0}$ is given by:

$$
\begin{aligned}
p_{1 \mid 0}= & \frac{27}{8} p\left(3, \frac{\gamma_{b}}{2}\right)-\frac{1}{8} p\left(3, \frac{\gamma_{b}}{6}\right)-\frac{81}{16} p\left(2, \frac{\gamma_{b}}{2}\right) \\
& -\frac{9}{16} p\left(2, \frac{\gamma_{b}}{6}\right)+\frac{81}{16} p\left(1, \frac{\gamma_{b}}{2}\right)-\frac{27}{16} p\left(1, \frac{\gamma_{b}}{6}\right) .
\end{aligned}
$$

For $N_{r}=4, p_{1 \mid 0}$ is given by:

$$
\begin{aligned}
p_{1 \mid 0}= & \frac{81}{16} p\left(4, \frac{3 \gamma_{b}}{8}\right)+\frac{1}{16} p\left(4, \frac{\gamma_{b}}{8}\right)-\frac{81}{8} p\left(3, \frac{3 \gamma_{b}}{8}\right) \\
& +\frac{3}{8} p\left(3, \frac{\gamma_{b}}{8}\right)+\frac{405}{32} p\left(2, \frac{3 \gamma_{b}}{8}\right)+\frac{405}{32} p\left(1, \frac{3 \gamma_{b}}{8}\right) \\
& +\frac{45}{32} p\left(2, \frac{\gamma_{b}}{8}\right)-\frac{135}{32} p\left(1, \frac{\gamma_{b}}{8}\right) .
\end{aligned}
$$

As is the case for $N_{r}=1$, the probability of bit error is given by:

$$
P_{b}<p_{15 \mid 0}+4 p_{1 \mid 0}+3 p_{3 \mid 0}
$$

4.3. BER Performance of MIMO-CDMA Using Parity-BitSelected Spreading. The upper bounds on the BER performance of MIMO-CDMA employing parity-bit-selected spreading that were derived in the previous subsections are shown in Figure 3. We have also simulated the BER performance using the same assumptions that were made in the derivation of the equations.

We see from Figure 3 that the upper bound is tight as $E_{b} / N_{o}$ increases as we would expect. This is due to the probability of the intersection of events (the distance between the received vector and desired vector is greater than two or more incorrect vectors) tends towards zero as $E_{b} / N_{o}$ increases. 


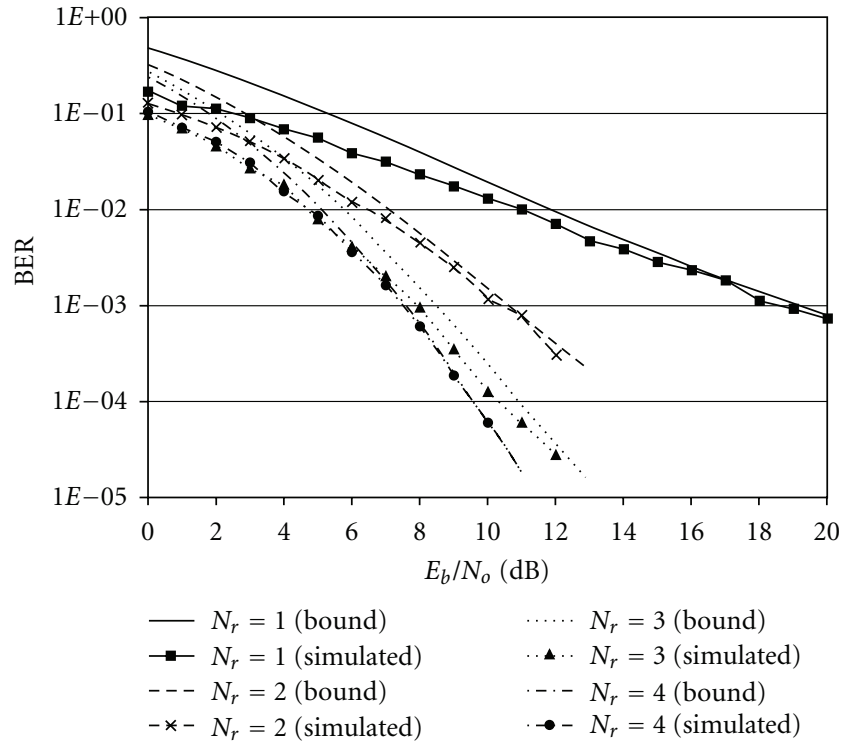

FIGURE 3: Comparison of BER upper bound to simulated performance of MIMO-CDMA employing parity-bit-selected spreading for $N_{t}=4$ and $N_{r}=1,2,3$, and 4 .

We also noticed that as $E_{b} / N_{o}$ increases, $P_{b}$ of (25) and (40) tends towards $p_{15 \mid 0}$ of (14) and (29), respectively. This means that as $E_{b} / N_{o}$ increases, the message being inverted is the most likely detection error. In other words, the most likely error is to detect the other message from the same coset. Let us consider the coset $\{0000,1111\}$. If we were to place 1111 in a different coset, for $N_{r}=1$, the distance squared $d_{15,0}^{2}$ would decrease to $2 E_{b}\left|\alpha_{11}+\alpha_{21}+\alpha_{31}+\alpha_{41}\right|^{2}$, thus increasing the probability of this error. If we were to include other messages in this coset, for example, 0011, then the distance squared between 0000 and 0011 would be $4 E_{b} \mid \alpha_{31}+$ $\left.\alpha_{41}\right|^{2}$ which is less than $d_{15,0}^{2}$, making it the dominant error. Thus, the pairing of a message with its one's complement is the optimum coset pairing with respect to the BER for this scheme.

It can be shown that the BER of a conventional singleuser MIMO-CDMA system operating in frequency nonselective Rayleigh fading has a probability of bit error given by:

$$
P_{b}=p\left(N_{r}, \frac{1}{N_{r}} \frac{E_{b}}{N_{o}}\right),
$$

when the fading on the different links is independent and the receiver has perfect knowledge of the channel gains. The conventional system assigns each antenna a unique spreading waveform, and the set of $\mathrm{Nt}$ waveforms are mutually orthogonal. From the above discussion, we know that as $E_{b} / N_{o}$ tends towards infinity, the probability of bit error for a single-user MIMO-CDMA system using the parity-bit-selected spreading technique has a bit error rate of

$$
P_{b}=p\left(N_{r}, \frac{N_{t}}{N_{r}} \frac{E_{b}}{N_{o}}\right) .
$$

Therefore, the MIMO-CDMA system employing the paritybit-selected spreading technique provides an asymptotic gain of $10 \log \left(N_{t}\right)$ over the conventional MIMO-CDMA system under the conditions discussed in this paper. As $E_{b} / N_{o}$ increases, the actual gain approaches but is less than $10 \log \left(N_{t}\right)$. This gain can be traded off against additional users accessing the common channel.

\section{Conclusion}

In this paper, we derived an analytical expression for the BER of a single user MIMO-CDMA system employing the paritybit-selected spreading technique in frequency nonselective Rayleigh fading under the assumptions of independently fading links and known channel gains. The results obtained from the analytical expression were compared against the simulated BER performance of the same system. The comparison shows that the derived upper bound is very tight to the simulated performance for moderate to high levels of signal to noise ratio. The expression also allows us to determine that for MIMO-CDMA employing paritybit-selected spreading, the most likely error results in an inversion of the message. This then further allowed us to determine that parity-bit-selected spreading provides an asymptotic gain of $10 \log \left(N_{t}\right)$ compared to conventional MIMO-CDMA. This gain can be traded off against additional users, thus increasing the spectral efficiency of MIMOCDMA systems at an expense of increased transmitter and receiver complexity.

\section{References}

[1] I. Koutsopoulos, U. C. Kozat, and L. Tassiulas, "Dynamic resource allocation in CDMA systems with deterministic codes and multirate provisioning," IEEE Transactions on Mobile Computing, vol. 5, no. 12, pp. 1780-1792, 2006.

[2] S. Verd, Multiuser Detection, Cambridge University Press, Cambridge, UK, 1998.

[3] A. J. Viterbi, "When not to spread spectrum-a sequel," IEEE Communications Magazine, vol. 23, no. 4, pp. 12-17, 1985.

[4] D. Haccoun, S. Lefrancois, and E. Mehn, "An Analysis of the CDMA capacity using a combination of low rate convolutional codes and PN sequence," in Proceedings of the Canadian Conference on Electrical and Computer Engineering (CCECE '96), vol. 1, pp. 32-35, Calgary, Canada, May 1996.

[5] Y. F. M. Wong and K. B. Letaief, "Concatenated coding for DS/CDMA transmission in wireless communications," IEEE Transactions on Communications, vol. 48, no. 12, pp. 19651969, 2000.

[6] R. D. Cideciyan, "Concatenated reed-solomon/convolutional coding for data transmission in CDMA-based cellular systems," IEEE Transactions on Communications, vol. 45, no. 10, pp. 1291-1303, 1997.

[7] Z. Guo and K. B. Letaief, "Performance of multiuser detection in multirate DS-CDMA systems," IEEE Transactions on Communications, vol. 51, no. 12, pp. 1979-1983, 2003.

[8] A. Duel-Hallen, J. Holtzman, and Z. Zvonar, "Multiuser detection for CDMA systems," IEEE Personal Communications, vol. 2, no. 2, pp. 46-58, 1995.

[9] K. Deng, Q. Yin, L. Ding, and Z. Zhao, "Blind channel estimator for V-BLAST coded DS-CDMA system in frequencyselective fading environment," in Proceedings of the 58th IEEE 
Vehicular Technology Conference (VTC-Fall '03), vol. 1, pp. 458-462, Orlando, Fla, USA, October 2003.

[10] S. Sfar and K. B. Letaief, "Layered group detection for multiuser MIMO wireless CDMA systems," IEEE Transactions on Wireless Communications, vol. 5, no. 9, Article ID 1687748, pp. 2305-2311, 2006.

[11] S.-M. Tseng, "Sequential detection for multiuser MIMO CDMA systems with single spreading code per user," IEEE Transactions on Wireless Communications, vol. 8, no. 7, pp. 3492-3497, 2009.

[12] W. Choi and J. G. Andrews, "Spatial multiplexing in cellular MIMO-CDMA systems with linear receivers: outage probability and capacity," IEEE Transactions on Wireless Communications, vol. 6, no. 7, pp. 2612-2621, 2007.

[13] M. Dohler, S. McLaughlin, D. Laurenson, M. Beach, C. M. Tan, and A. H. Aghvami, "Implementable wireless access for B3G networks: part I. MIMO channel measurement, analysis, and modeling," IEEE Communications Magazine, vol. 45, no. 3, pp. 85-92, 2007.

[14] M. Dohler, S. McLaughlin, and A. H. Aghvami, "Implementable wireless access for B3G networks: part II. MIMO receiver architectures," IEEE Communications Magazine, vol. 45, no. 3, pp. 93-97, 2007.

[15] C. D'Amours, "Parity bit selected spreading sequences: a block coding approach to spread spectrum," IEEE Communications Letters, vol. 9, no. 1, pp. 16-18, 2005.

[16] C. D'Amours and J.-Y. Chouinard, "Parity bit selected and permutation spreading for CDMA/MEMO systems in frequencynonselective rayleigh fading channels," in Proceedings of the 65th IEEE Vehicular Technology Conference (VTC '07), pp. 1475-1479, Dublin, Ireland, April 2007.

[17] C. D’Amours and A. O. Dahmane, "Spreading code assignment strategies for MIMO-CDMA systems operating in frequencyselective channels," EURASIP Journal on Wireless Communications and Networking, vol. 2009, Article ID 839424, 13 pages, 2009.

[18] A. O. Dahmane and C. D’Amours, "Spreading strategies for MIMO-CDMA in presence of channel estimation errors and spatial correlation," in Proceedings of the 69th IEEE Vehicular Technology Conference (VTC '09), pp. 1-5, Barcelona, Spain, April 2009.

[19] J. G. Proakis and M. Salehi, Digital Communications, McGrawHill, New York, NY, USA, 5th edition, 2008. 

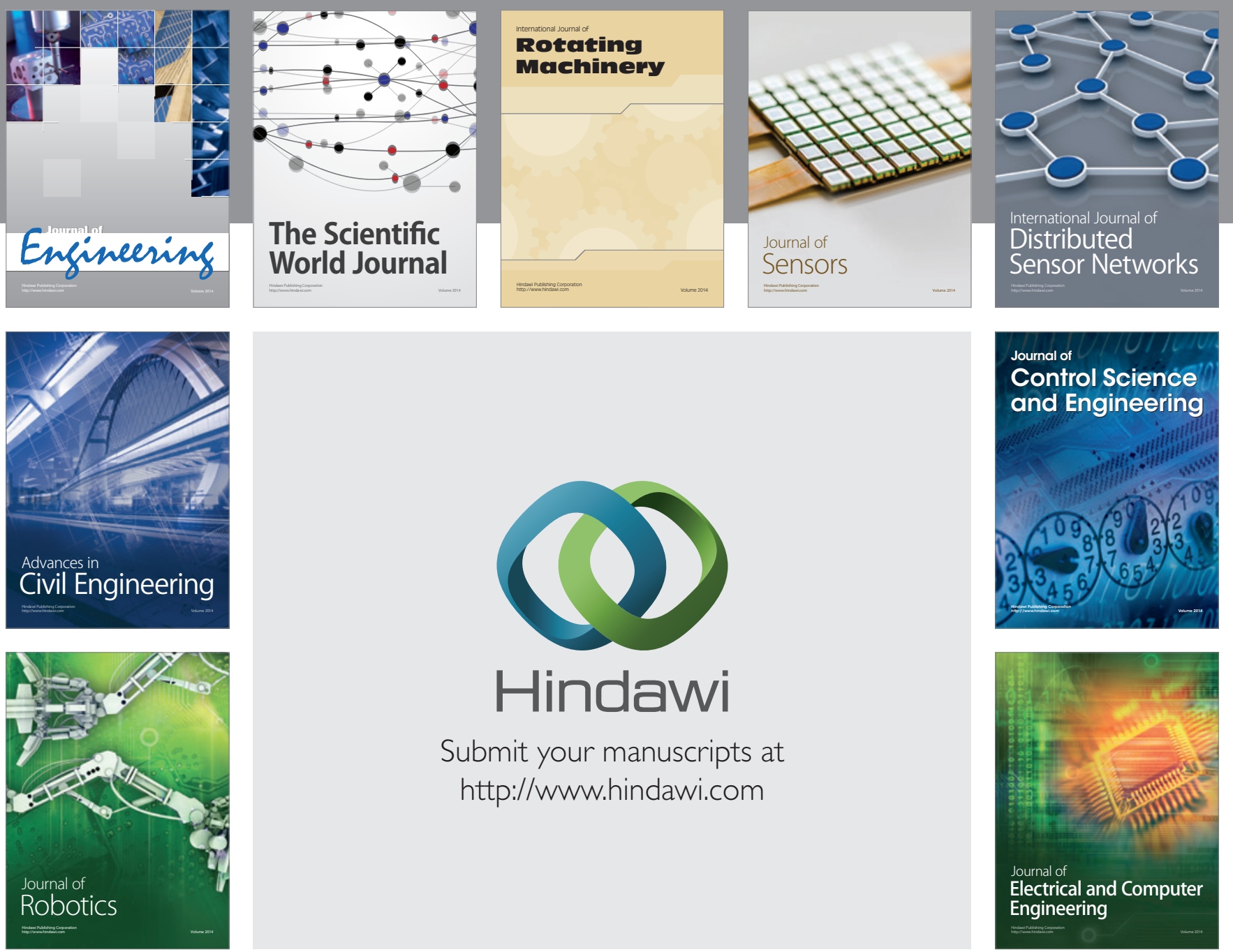

Submit your manuscripts at

http://www.hindawi.com
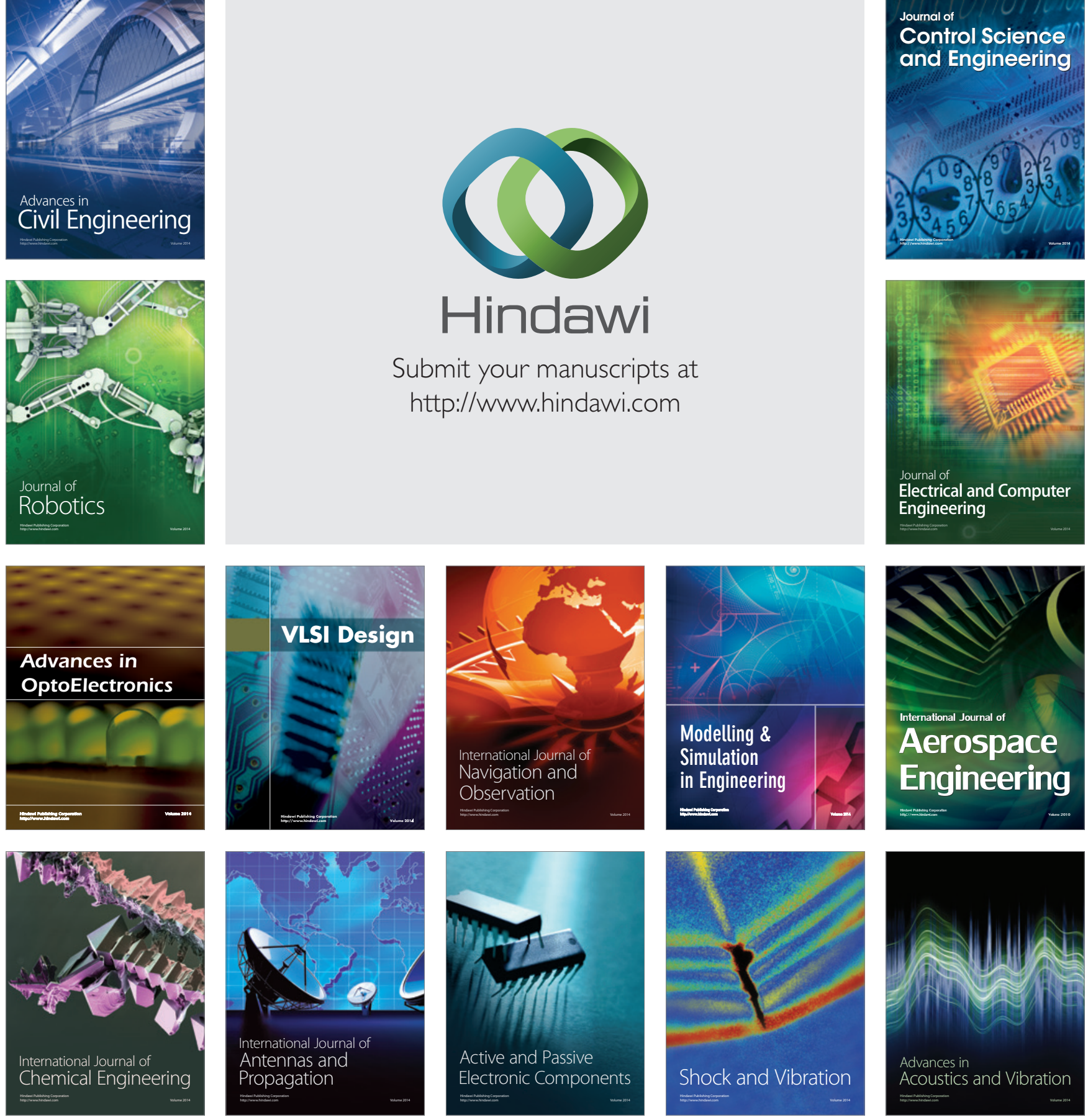